\title{
НЕЗАВИСЯЩИЙ ОТ СТРОЕНИЯ СРЕДЫ ОПЕРАТОР УДАЛЕНИЯ КРАТНЫХ ВОЛН
}

Орлов Ю.А.

(Институт нефтегазовой геологии и геофизики им. А.А. Трофижука СО РАН)

Резюме. Для подавления кратных волн предлагается новый подход, основанный на сравнении двух постановок прямой задачи для сред с одинаковыми нижкнимх и разными верхними полупространствами. В первой среде верхнее полупространство является однородной средой с прозрачной границей раздела полупространств, а во второй - границей нгжнего полупространства является свободная граница, приводящая к отражению и возникновению кратных волн. Из сопоставления этих двух задач дается вывод решения для оператора удаления кратных волн, независящего от строения среды.

Рассмотрим постановку прямой динамической задачи дпя двух сред с одинаковьми ншжними полупространствами. В первой среде верхнее полупространство однородно и опсутствуют перепады упругих параметров на гранище полупространств то есть эта гранхца прозрачна. Во второй среде верхняя гранища полупространства является свободной. Считаем, что группированием источников или соответствующим суммированием сейсмограмм от разных источников в обенх средах формируется одинаковая плоская падающая гармоническая волна, распространяющаяся вниз с амплитудой $u_{0}(\omega)$, где $\omega$ - пастота. Система наблюдения ограничена и дискретна.

Целью настоящей работы является получение выражений для прямого и обратного оператора, который связывает зарегистрированные поля для этих двух сред.

Для первой среды обозначим через $U \uparrow(\omega, k, \xi)$ результат дискретного преобразования Фурье по пространственной переменной от поля, зарегистрированного на гранище полупространств, где $k$ и $\xi$ целые числа, однозначно определяющие волновые векторы падающей и регистрируемой волн.

Результат дискретного преобразования Фурье от поля, зарегистрированного на свободной границе, для второй среды обозначим $U(\omega, \xi, k)$. Для этой среды на свободной границе необходимо поставить соответствующее граничное условие. Для SH-волны такое условие приводит к отражению волн с коэффициентами: отражения $\chi=1$ и конверсии $\eta=2$. Для других типов волн или при регистрации вблизи свободной границы эти коэффициенты зависят от частоты и угла падения.

Для получения связи между волновыми полями этих двух сред используется свойство линейности уравнение упругости (акустики) и трактовка волн, отраженных от свободной поверхности, как волн, формируемых виртуальными источниками. Для того чтобы привести зарегистрированное поле для второй среды к полю для первой среды, нужно выполнить следующие преобразования:

$$
\mathbf{U} \uparrow(\omega)=u_{0}(\omega) \mathbf{Q}(\omega) \mathbf{W}(\omega)
$$

$$
\mathbf{Q}(\omega)=(\mathbf{E}+\mathbf{W}(\omega))^{-1}
$$

Квадратные матрищы в выражениях (1) и (2) имеют следующие компоненты: $\mathbf{U} \uparrow(\omega)$ $U \uparrow(\omega, k, \xi) ; \mathbf{W}(\omega)=\chi U(\omega, \xi, k) / \eta u_{0}(\omega) ;$ и E - единичная матрица.

В результате такой операции поле, зарегистрированное на свободной границе, привели к полю, регистрируемому на прозрачной границе с однородным верхним полупространством, которое не содержит кратных волн. Выражение (2) можно трактовать как оператор удаленхя кратных волн. Важно подчеркнуть, что эти преобразования осуществляется цля стационарного случая и не содержат никаких предположений и сведений о строения среды. Для построения ОУКВ необходимо знать только спектральную характеристику источника. ОУКВ является нелинейным фильтром (по временной и пространственной частоте) как это и было предсказано*.

В морской сейсмике при регистрации поля датчиками давления на глубине $h_{R}$ и гри расположении источников на глубине $h_{S}$ компоненты матрицы $\mathbf{W}(\omega)$ имеют вид

$$
\bar{W}(\omega, \xi, k)=\chi \frac{\bar{U}(\omega, \xi, k)}{\left(1+\chi \exp \left(2 h_{R} k_{z}\right)\right) u_{0}(\omega)} \exp \left(-\left(h_{s}-h_{R}\right) \xi_{z}\right)
$$

, где $\xi_{z}$ и $k_{z}$ - вертикальные компоненты волновых векторов падающей и регистрируемой волн соответственно. В этом выражения учтено изменение формы волны, обусловленное волной спутником.

Запись операции удаления кратных волн в пространственных координатах имеет такой же вид как и (1-2), но индексы соответствуют номерам пунктов приема и возбуждения, координаты которых должны быть одинаковы. Для SH-волны ОУКВ в пространственных координатах применим и для свободной границы с рельефом, для других типов волн возникает проблема, связанная с зависимостью коэффициентов отражения от угла падения.

В заключение отметкм, что эффективность применения ОУКВ показана на синтетических данныг и дано сравнение результатов работы ОУКВ и реверберационного фитьтра

Работа поддержана межсисчиплинарным uнтеграичоннын проектож СО РAH (No 26).

*Гольдин С.В. Линейные преобразования сейсмических ситналов. М.: «Недра», 1974, - 351 с. 